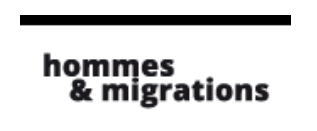

\section{Hommes \& migrations}

Revue française de référence sur les dynamiques

migratoires

$1296 \mid 2012$

Le Mexique dans les migrations internationales

\title{
La nouvelle loi sur la migration au Mexique ou "Le bon, la brute et le truand"
}

\section{Primitivo Rodríguez Oceguera}

Traducteur : Jean Hennequin

\section{OpenEdition}

\section{Journals}

Édition électronique

URL : http://journals.openedition.org/hommesmigrations/1511

DOI : 10.4000/hommesmigrations. 1511

ISSN : 2262-3353

Éditeur

Musée national de l'histoire de l'immigration

Édition imprimée

Date de publication : 1 mars 2012

Pagination : 64-72

ISSN : 1142-852X

Référence électronique

Primitivo Rodríguez Oceguera, "La nouvelle loi sur la migration au Mexique ou "Le bon, la brute et le truand" », Hommes \& migrations [En ligne], 1296 | 2012, mis en ligne le 01 mars 2014, consulté le 10 décembre 2020. URL : http://journals.openedition.org/hommesmigrations/1511 ; DOI : https:// doi.org/10.4000/hommesmigrations.1511 


\section{La nouvelle loi sur la migration au Mexique ou "Le bon, la brute et le truand"}

Par Primitivo Rodríguez Oceguera, doctorant en histoire, université de Chicago, membre fondateur de la Coalition pour les droits politiques des Mexicains à l'étranger

Pays d'origine, de destination, de passage et de retour des migrants, Le Mexique vient de se doter en 2011 d'une nouvelle loi sur la migration.

Si la volonté de mieux encadrer les flux de populations en

provenance ou à destination des États-Unis peut paraître louable en termes de défense des migrants, elle traduit surtout l'application

d'une stratégie sécuritaire sous la houlette du voisin américain.

Pour paraphraser le célèbre western de Sergio Leone :

"Le monde se divise en deux catégories : ceux qui ont un pistolet chargé et ceux qui sont contrôlés... toi, tu es contrôlé". 
La loi sur la migration, approuvée en avril 2011 par le congrès mexicain, a fait renaître les craintes concernant ceux qui, arrivant d'autres pays, ont des façons différentes de voir le monde, professent parfois des credo contraires à la religion catholique, parlent d'autres langues et ont la peau d'une autre couleur.

Les succès remportés par les colons texans au cours de leur mouvement d'indépendance des années 1836-1837, puis la perte de la moitié du territoire mexicain à la suite de la guerre contre les États-Unis, ont légué au Mexique un héritage traumatique : la méfiance envers les immigrants, surtout si ceux-ci sont anglo-saxons et protestants. Par ailleurs, le développement accéléré des États-Unis, à la fois rural et urbain, a donné au Mexique une autre raison de poids qui l'a conduit à négliger l'immigration : s'agissant d'attirer les migrants, il n'y avait pas moyen de concurrencer le voisin du Nord. Pis encore, la demande croissante de travailleurs étrangers de la part des ÉtatsUnis a fait du Mexique le pourvoyeur naturel de main-d'ceuvre bon marché puis, à partir des années quatre-vingt-dix, d'une main-d'œuvre qualifiée, urbaine, ayant un niveau d'instruction supérieur à l'éducation primaire, ainsi que de techniciens et de professionnels. En matière de migration, de même que dans bien d'autres domaines de la vie nationale, les États-Unis ont mis en évidence les limites et les défaillances du "rêve mexicain", contrastant avec l'essor et la suprématie du "rêve américain". Après la guerre de 1846-1848 contre le Mexique et la proclamation officielle de la Confédération canadienne en 1867, les États-Unis ne connurent plus aucun problème de sécurité le long de leurs frontières terrestres. Ce privilège sans précédent dans l'histoire moderne des grandes puissances mondiales commença à s'effondrer vers le milieu des années quatre-vingt-dix, lorsque Washington entreprit la création d'un mur d'acier à la frontière du Mexique afin d'empêcher le passage des migrants, et culmina avec les strictes mesures de sécurité frontalière imposées par les États-Unis en réponse aux actes terroristes du 11 septembre 2001. C'est dans ce contexte, et après l'avoir cantonnée durant trente-sept ans au sein de la loi générale sur la population, que le congrès a promulgué une nouvelle loi sur la migration, grâce à laquelle le Mexique serait censé avoir récupéré son autorité morale face aux États-Unis et occuperait désormais une position d'avant-garde au niveau international en matière de vision et de gestion du phénomène migratoire. Qu'en est-il en réalité ?

\section{"Le bon" dans la nouvelle loi mexicaine sur la migration(1)}

Le meilleur de cette loi se trouve dans son exposé des motifs. Les législateurs établissent clairement que : "La loi a pour objet de faire respecter à tout instant et en tout point du territoire national la dignité ainsi que les droits des migrants, quelle que soit leur 
condition migratoire, en prêtant la plus grande attention aux personnes les plus vulnérables, telles que les enfants et les femmes sans papiers. Désormais, le traitement à réserver aux migrants se fondera sur les lois mexicaines, ainsi que sur les documents internationaux souscrits et ratifiés par le Mexique, tels que la Convention internationale sur la protection des droits de tous les travailleurs migrants et des membres de leur famille, la Convention internationale sur l'élimination de toutes les formes de discrimination raciale et les Principes régionaux d'assistance aux enfants et adolescents migrants non accompagnés en cas de rapatriement. L'asile relève d'une autre loi mexicaine. La loi possède un caractère intégral. Elle correspond à la caractéristique du Mexique en tant que pays d'origine, de destination, de passage et de retour des migrants. Le contenu de la loi a été établi en tenant compte des points de vue d'universitaires, de représentants d'organisations sociales, de membres du gouvernement, de fonctionnaires et de législateurs, et en se

Au vu de l'expérience de certains pays tels que les États-Unis ou le Mexique lui-même, on peut affirmer que les stratégies consistant à fermer hermétiquement les frontières aux sans-papiers, afin d'en faire la "première ligne" de défense de la société et de la nation, se sont avérées contre-productives. fondant sur l'étude des lois et des politiques migratoires de l'Argentine, du Brésil, de l'Espagne, de la France, des États-Unis, ainsi que d'autres pays."

De même, l'exposé des motifs définit les douze principes "sur lesquels doit se fonder la politique migratoire de l'État mexicain", à savoir : approche intégrale ; responsabilité partagée ; respect des droits des migrants; hospitalité et solidarité internationale ; aide à la mobilité internationale des personnes ; complémentarité des marchés du

travail avec les pays de la région ; équité de traitement envers tous les individus, aussi bien nationaux qu'étrangers ; reconnaissance des droits acquis ; unité familiale et intérêt suprême de l'enfant et de l'adolescent ; intégration sociale et culturelle entre nationaux et étrangers ; aide au retour au territoire national et à la réinsertion sociale des émigrés mexicains et de leurs familles. En matière de reconnaissance des droits, il convient de mentionner tout particulièrement les articles 8 à 15, lesquels établissent que tout migrant a droit à l'accès à l'éducation, à la santé, à l'état civil, ainsi qu'au système d'administration de la justice. Ces articles reconnaissent également le droit à l'unité familiale, la personnalité juridique des migrants, ainsi que leur intégration à la société mexicaine en ce qui concerne leur identité et leur diversité ethnique et culturelle.

À la vue de tels contenus, la loi sur la migration semble constituer un document faisant du Mexique le paradis de tout migrant, aussi bien national que ressortissant d'un autre pays. Mais à y regarder de plus près... 


\section{"La brute..." ou les véritables intentions des législateurs}

Malgré l'exposé des motifs servant d'introduction à cette nouvelle loi et en dépit des discours enthousiastes que prononcèrent les législateurs au moment de l'approuver, celle-ci présente un certain nombre de défauts qui, faute d'être corrigés, viendront annuler dans la pratique les bienfaits qu'elle comporte, ainsi que les avantages qu'elle accorde aux migrants. En voici quelques exemples.

\section{Migration et sécurité}

Bien qu'il soit affirmé à deux reprises que la loi a pour objet de protéger la sécurité humaine des migrants, le ton et le langage prévalents concernent la sécurité frontalière, la sécurité publique et la sécurité nationale. Tout porte à croire que l'allusion à la sécurité humaine des migrants n'a d'autre but que de faire de cette loi un document "politiquement correct". En réalité, le sens de cette loi, de même que l'objectif central que poursuit l'agence fédérale chargée de la mettre en ceuvre et de l'administrer, l'Institut national de la migration (INM), semblent bien être de renforcer les stratégies de lutte contre le trafic de drogues, contre d'éventuels réseaux terroristes et contre la délinquance organisée liée à la migration.

Mêler le thème de la migration à celui de la sécurité frontalière, publique et nationale, ne profite en rien aux migrants pas plus qu'à la société ou à l'État. Au vu de l'expérience de certains pays tels que les États-Unis ou le Mexique lui-même, on peut affirmer que les stratégies consistant à fermer hermétiquement les frontières aux sans-papiers, afin d'en faire la "première ligne" de défense de la société et de la nation, se sont avérées contre-productives : elles alimentent la peur, les préjugés et les abus envers les migrants, favorisent l'augmentation de la délinquance organisée et encouragent la corruption de la police et des autorités.

Même à supposer que les mesures qui ont été adoptées en vue de fermer les frontières des États-Unis et du Mexique aux sans-papiers aient effectivement contribué à réduire les flux de la migration clandestine, le coût qu'elles ont représenté en termes d'abus envers les migrants, de morts de ceux-ci, d'augmentation de la délinquance et de la corruption, s'avère fort élevé et extrêmement nocif pour la santé de la population et pour celle du pays.

\section{Migrants sans papiers en transit vers les États-Unis}

Les guerres civiles qui déchirèrent l'Amérique centrale au cours des années soixantedix et quatre-vingt, ainsi que l'intervention militaire de Washington à laquelle celles-ci donnèrent lieu, provoquèrent des flux migratoires massifs, notamment en 
direction des États-Unis. Ceci obligea le Mexique à renforcer les mesures de surveillance sur sa frontière Sud, sans toutefois se soucier plus que de raison des migrants originaires d'Amérique centrale en transit vers les États-Unis. Cependant, à la suite des actes terroristes du 11 septembre 2001, le Mexique a inscrit le thème du contrôle de la migration des sans-papiers à son agenda de sécurité. Un tel changement a porté gravement atteinte aux migrants sans papiers, ainsi qu'à l'autorité morale du Mexique et à son image à l'étranger.

À la poursuite du "rêve américain", les migrants en transit finissent par sombrer dans "l'enfer mexicain": abus, enlèvements, extorsions, traites, viols, tortures et assassinats de la part de la délinquance organisée, ainsi que de la part des agents de police et des fonctionnaires corrompus ou agissant de connivence avec les bandes criminelles.

Malgré cette tragédie humaine, la loi sur la migration, dans ses articles 40, 41 et 52, est ambiguë en ce qui concerne l'octroi de visas ou de laissez-passer aux sans-papiers se dirigeant vers les États-Unis. Cette ambiguité est encore accentuée par les articles 34 et 47, qui établissent que tout citoyen mexicain ou ressortissant étranger qui sort du pays doit le faire en suivant un itinéraire prédéterminé et muni des pièces d'identité correspondantes. Les sans-papiers qui se rendent aux États-Unis ne traversent pas le Mexique en suivant un itinéraire prédéterminé ni munis de visas, pas plus que ne le font les sans-papiers mexicains. La loi sur la migration est actuellement en cours de réglementation, tâche qui incombe au pouvoir exécutif. Celui-ci, profitant de l'ambiguïté qu'a laissé planer le congrès, pourrait fort bien octroyer un visa ou un laissez-passer aux migrants en transit, afin de remédier une fois pour toutes à l'extrême vulnérabilité à laquelle se trouvent exposés ces hommes et ces femmes.

\section{La courte vue des pouvoirs publics mexicains}

À en croire les législateurs qui l'ont approuvée, la nouvelle loi sur la migration possède un caractère intégral, c'est-à-dire qu'elle est censée répondre aux opportunités et aux défis propres au Mexique en tant que pays d'origine, de destination, de passage et de retour de migrants. Cependant, cette loi se réfère fort peu et, qui plus est, de façon très générale, aux mesures destinées à encourager l'immigration. Elle omet de spécifier certains points fondamentaux, tels que les objectifs et les critères de l'immigration, le profil et l'expérience professionnelle auxquels doivent satisfaire les migrants, leur composition par genre, les mesures d'encouragement susceptibles de les attirer, ainsi que les facilités qui leur seraient accordées afin qu'ils puissent acquérir la résidence ou la citoyenneté.

C'est pourquoi, loin d'être le fruit de politiques publiques, l'arrivée d'immigrants au Mexique continuera à être le résultat de décisions personnelles, familiales ou de groupe, de la part d'individus cherchant à s'établir dans le pays, ou de parents et amis 
qui y résident déjà. Dans un monde caractérisé par une mobilité humaine toujours croissante et une grande complexité des marchés de travail, la faible implication du Mexique afin d'attirer les migrants ne saurait en rien profiter au pays.

\section{Une diaspora mexicaine sous-estimée dans le développement national}

Un autre grand défaut, sans doute le plus grave, dont souffre la nouvelle loi sur la migration, est qu'elle néglige l'énorme potentiel, en matière d'épargne, d'investissement et de création d'entreprises, que représente la diaspora mexicaine pour créer un capital humain au Mexique et enrichir celui qui existe déjà.

Ainsi, les programmes d'échange d'étudiants, d'universitaires, de professionnels et de chefs d'entreprise mexicains avec leurs homologues étrangers, demeurent encore assez rares. Par ailleurs, en dehors du débat autour de la question de savoir si les envois d'argent contribuent ou non au développement, on ne parle guère de la façon dont pourrait se concrétiser l'une des aspirations que partagent des milliers de migrants : encourager les créations d'emploi et les investissements dans leurs communautés et régions d'origine.

Le jour où les lois et les politiques offriront à la diaspora mexicaine des facilités ainsi que des mesures d'encouragement comparables à celles dont bénéficient à l'étranger les investisseurs et les compagnies, le Mexique aura découvert une ressource bien plus précieuse que les traités de libre commerce qu'il a souscrits ou que l'exploitation du pétrole.

\section{Des sanctions envers les passeurs communautaires, les membres de la famille et les amis}

Aux termes de l'article 159, alinéa I, de la nouvelle loi sur la migration, "sera passible d'une peine d'emprisonnement allant de huit à seize ans [...] quiconque se livrera au trafic consistant à aider une ou plusieurs personnes à entrer dans un autre pays sans les pièces d'identité correspondantes, à des fins de lucre direct ou indirect'".

Bien qu'il vise à combattre le trafic de migrants, cet article finira non seulement par donner le coup de grâce aux passeurs connus et respectés dans leur communauté d'origine, mais aussi par encourager le monopole du transport de migrants sans papiers de la part de la délinquance organisée, qui seule disposera de l'argent nécessaire afin de créer des réseaux transnationaux, corrompre les agents de police et les fonctionnaires, payer les avocats.

Les graves sanctions prévues par la loi dissuaderont également les parents et amis des migrants désireux d'entrer aux États-Unis de les aider à réaliser leur projet, ne seraitce qu'en échange de quelque argent. 
Tant que Washington ne mettra pas en place une réforme migratoire destinée à régulariser la demande de travailleurs internationaux et ne réduira pas les délais nécessaires à l'obtention de visas de regroupement familial, d'importants contingents de migrants sans papiers continueront à affluer vers ce pays. Pour les mêmes raisons, le gouvernement mexicain devrait s'attacher à poursuivre et à punir la délinquance organisée qui se consacre à enlever, extorquer, violer, voire assassiner les migrants sans papiers et, en même temps, à cesser de mettre sur un pied d'égalité les membres de telles bandes et les passeurs communautaires, les parents et amis de migrants sans papiers qui les aident à franchir la frontière des États-Unis.

\section{De l'instrumentalisation du Mexique par les États-Unis : ".... et le truand"}

Avant l'ouverture des négociations concernant l'Accord de libre-échange nordaméricain (Aléna), le Mexique était encore considéré par les pays d'Amérique latine et des Caraïbes comme un "frère aîné", représentant "la frontière latino-américaine" par rapport aux États-Unis. Après la signature de l'Aléna en 1993 et après avoir souscrit en 2005 le Partenariat pour la sécurité et la prospérité de l'Amérique du Nord (PSP), le Mexique a perdu son rôle - réel ou symbolique - d'interlocuteur entre les nations situées au sud du Río Bravo et le "colosse du Nord". Il est désormais considéré comme un pays qui a fini par céder à son destin manifeste: celui d'être, de fait, le héraut des stratégies commerciales et sécuritaires des États-Unis.

\section{Faire le "sale travail" des États-Unis en matière migratoire}

L'image d'un Mexique qui prend de plus en plus ses distances à l'égard de ses racines et de sa vocation latino-américaines doit son origine à l'adoption de politiques de contrôle des migrants d'Amérique centrale en transit vers les États-Unis, notamment à la suite des mesures de sécurité frontalière draconiennes imposées par Washington, au niveau mondial et régional, en réponse aux actes terroristes du 11 septembre 2001. L'Initiative de Mérida, accord de sécurité signé en 2007 par les présidents George W. Bush et Felipe Calderón, a consolidé la responsabilité du Mexique dans la lutte contre le trafic de drogues, le terrorisme et la migration des sans-papiers. La question pourrait être posée de savoir si la nouvelle loi sur la migration répond à des besoins et à des intérêts proprement mexicains ; quoi qu'il en soit, il ne fait aucun doute qu'elle constitue une pièce fondamentale des stratégies sécuritaires mises en place par l'Initiative de Mérida. 
Il n'est donc pas surprenant que le Mexique, considéré naguère par les pays latinoaméricains et les Caraïbes comme le défenseur des droits des migrants face à Washington, en est venu à être considéré comme le laquais chargé de faire, pour le compte des États-Unis, le "sale travail" contre les migrants d'Amérique centrale. En termes de contrôle migratoire, la frontière des États-Unis ne commence plus au Texas ou en Californie, mais au Chiapas et au Tabasco, si bien que la redoutable migra $^{(2)}$ américaine est devenue une simple Armée du salut comparée aux nombreuses et féroces migras mexicaines : agents de la police nationale, municipale et des différents États de la fédération, agents des services de migration et unités des forces armées qui opèrent non seulement aux frontières, mais sur l'ensemble du territoire national.

\section{La perte de toute autorité morale dans la défense des migrants}

Dès la fin du $\mathrm{XX}^{\mathrm{e}}$ siècle s'est répandue à travers l'Union européenne et les États-Unis la nouvelle de cette tragédie humaine que représente le passage sur le territoire mexicain de milliers de sans-papiers tous les ans. Médias imprimés et audiovisuels, documentaires, films et témoignages des migrants eux-mêmes ont fait connaître l'existence de cette douloureuse réalité. Cependant, l'assassinat à San Fernando (État du Tamaulipas), en octobre 2010, de 72 migrants en provenance d'Amérique centrale et d'Amérique du Sud, aux mains, semblerait-il, d'un cartel de la drogue, est venu révéler la terrible dégradation de la sécurité humaine au Mexique, ainsi que l'extrême vulnérabilité à laquelle sont exposés ces femmes et ces hommes sans papiers.

Depuis plus d'une dizaine d'années déjà le Mexique a perdu toute autorité morale pour exiger le respect des droits de ses citoyens à l'étranger. Toutefois, après le massacre de San Fernando il ne semble avoir d'autre choix que d'adopter des lois et des politiques d'ouverture favorisant une nouvelle rencontre de l'État avec sa diaspora, ainsi qu'avec les migrants en provenance d'autres pays.

\section{Conclusion}

Pour l'instant, le Mexique ne se trouve encore engagé qu'à mi-chemin sur la voie de sa reconnaissance en tant que pays d'origine, de destination, de passage et de retour de migrants. En ce sens, la loi sur la migration ne constitue qu'un moyen terme : tantôt elle semble être la photographie restaurée de ce Mexique jadis fier de sa généreuse solidarité envers les femmes et les hommes à la recherche d'un travail, d'une terre d'exil ou de refuge ; un pays à la fois renouvelé et enrichi par tous ceux qui en ont fait leur foyer pour un instant ou leur seconde patrie pour toute la vie. Tantôt, au 
contraire, elle semble dévoiler un Mexique plein de craintes et de méfiance envers tous ceux qui ne sont pas nés sur son sol, qui viennent de mondes différents, un Mexique qui n'oublie pas les énormes territoires qui lui ont été enlevés par les migrants anglo-saxons, ni la sueur et les larmes que ses enfants ont versées dans les campagnes et les villes américaines, un Mexique qui tonne contre son voisin du Nord tout en lui obéissant, qui salue comme des frères les travailleurs migrants d'Amérique centrale tout en les outrageant et en les harcelant.

Quoi qu'il en soit, le Mexique ne saurait sortir de ce genre d'imbroglio que grâce à la diaspora mexicaine, ainsi qu'à toutes les femmes et tous les hommes qui, venus d'autres pays pour travailler temporairement sur son sol,y résider ou l'utiliser comme lieu de passage vers les États-Unis, ont d'ores et déjà joué un rôle de premier plan dans cette tâche privilégiée. L'avenir du Mexique en tant que nation ouverte et prospère se présente sous le visage du migrant.

\section{Traduit de l'espagnol par Jean Hennequin}

\section{Notes}

1. Pour avoir accès au texte de la loi sur la migration, ainsi qu'à l'exposé des motifs correspondant, taper sur le moteur de recherche Google la requête "Minuta Ley de migración 29 de abril de 2011" (entre guillemets).

Le premier résultat correspond à la page électronique "Huellas Mexicanas", qui est celle qu'il convient de consulter : y est reproduit le texte complet de la minute du Sénat, approuvée à l'unanimité et sans aucune modification par la Chambre des députés, avant de devenir la loi actuelle sur la migration.

Par ailleurs, la requête "Diario Oficial de la Federación 25 de mayo 2011", toujours sur Google, permet d'accéder au texte officiel de la loi, qui ne comprend pas l'exposé des motifs : http://dof.gob.mx/nota_detalle.php? codigo=5190774\&fecha=25/05/2011.

2. La migra: nom populaire donné par les Latino-Américains à la police migratoire des États-Unis [N. du T.]. 